\title{
Captifs de chaînes électroniques
}

Des entraves électroniques en guise de chaînes aux pieds sont la promesse d'une peine plus humaine, d'une meilleure sécurité et d'une réduction des coûts, et ce par le biais d'une méthode diminuant le personnel de surveillance carcérale et économisant les séjours coûteux en prison. Les experts en justice et en médecine parlent ici le même langage. En effet, sur le plan technique, aucune différence ne les sépare, qu'il s'agisse de données biométriques, de surveillance télévisuelle, de dossiers de patients, de suivi des cures de bien-être, de sites de santé, de systèmes de positionnement mondial (GPS) ou de techniques de radio-identification (RFID). Etant donné les sommes investies, les fabricants des technologies de l'information mettent les politiques sous pression. A les en croire, nous sommes un pays en voie de développement et le Danemark, l'Autriche et nombre d'autres sont des modèles à citer en exemple: www.sundhed.dk offre un réseau central pour tous les hôpitaux, laboratoires, spécialistes et patients; Vienne a déjà introduit la carte de patient et planifie la carte électronique de santé; l'Hôpital universitaire de Nice a équipé ses patients, appareils et médecins de dispositifs de radio-identification au poignet; plusieurs pays expérimentent un système de transmission de données corporelles par téléphone mobile; les biobanques, par l'intégration des données, font espérer des diagnostics, des traitements et des mesures prophylactiques plus efficaces.

La numérisation du système de santé est aussi en pleine expansion en Suisse. Vu l'asymétrie considérable entre utilisateurs et fabricants, les avantages de cette évolution sont plus évidents que ses risques. La médecine, l'administration et la politique sont dépassées par les progrès éclatants des prestations électroniques. Tous les transferts de données laissent des traces: ils peuvent se conserver, s'associer et se consulter sans peine. Ils donnent un profil virtuel et se prêtent à n'importe quel objectif. Ce ne sont pas les consommateurs qui nous avertissent des dangers que ces techniques représentent, mais plutôt les spécialistes de la protection des données et les instituts d'évaluation des choix technologiques. Ceux-ci nous mettent en garde contre la «servitude insidieuse» que représente le fait de devoir régir dans la loi l'enregistrement des données de télécommunication à conserver, la localisation exacte des utilisateurs de téléphones portables, le devoir d'identification des ordinateurs privés, l'abolition des courriels anonymes ou les investigations secrètes en ligne. Le système en place est un magasin libre service pour les amateurs de marketing, les fouineurs de l'Etat et les criminels de toutes sortes. La mise en danger de la sphère privée est très fortement sous-estimée par rapport aux avantages immédiats et au confort technique. Même les experts de l'image et du traitement de l'information sont convaincus que la protection des données est un domaine sensible qui, dans le secteur de la santé, n'est pas résolu aujourd'hui.

Lors de la $1^{\text {re }}$ journée européenne sur le thème de la protection des données, ce printemps à Zurich, on a parlé de l'ignorance du citoyen à ce sujet, un déficit que le SwissSecurityDay 2007 désire combler avec des informations sur la sécurité des données, la protection anti-virus, les parefeu, l'actualisation des logiciels et le comportement général à adopter. Les utilisateurs privés, les écoles, les administrations et les entreprises seront désormais informés chaque année des dangers de l'internet. Un groupe européen pour l'évaluation des conséquences de la technologie, dont fait partie la firme TA-SWISS, a publié pour la première fois un rapport commun sur le thème «ICT and Privacy in Europe» [1]. Le document en anglais a paru en octobre 2006. Il livre un «cours de rattrapage» indispensable pour toutes celles et ceux qui s'intéressent à la santé par voie électronique et aux autres utilisations quotidiennes de l'informatique. Les technologies et les termes techniques sont brièvement et bien expliqués. Le cours donne un aperçu exhaustif des chances et des risques des nouvelles technologies dans des domaines comme la sécurité et l'accessibilité des données, les interactions sociétales, les prestations de confort, les bénéfices économiques, la gouvernance électronique et les soins de santé. La Suisse a livré un chapitre détaillé sur la cybersanté (e-health). Le rapport cite huit défis concrets et se termine par des recommandations à l'intention des gouvernements. Les auteurs estiment que la difficulté essentielle réside dans une relation déséquilibrée entre respect de la sphère privée et conscience du problème. Ils souhaitent qu'on adopte une attitude préventive. Les développements captivants du futur sont aussi une manière de nous enchaîner.

Erhard Taverna countries. Bruxelles: EPTA; 2006. 117 pages. www.ta-swiss.ch.

European Parliamentary Technolin Europe. Experiences from technology assessment of ICT and Pri- 